PROCEEDINGS OF THE

AMERICAN MATHEMATICAL SOCIETY

Volume 137, Number 11, November 2009, Pages 3553-3562

S 0002-9939(09)09990-0

Article electronically published on June 25, 2009

\title{
ALMOST UNIVERSAL TERNARY SUMS OF TRIANGULAR NUMBERS
}

\author{
WAI KIU CHAN AND BYEONG-KWEON OH
}

(Communicated by Wen-Ching Winnie Li)

\begin{abstract}
For any integer $x$, let $T_{x}$ denote the triangular number $\frac{x(x+1)}{2}$. In this paper we give a complete characterization of all the triples of positive integers $(\alpha, \beta, \gamma)$ for which the ternary sums $\alpha T_{x}+\beta T_{y}+\gamma T_{z}$ represent all but finitely many positive integers, which resolves a conjecture of Kane and Sun.
\end{abstract}

\section{INTRODUCTION}

In 1796 Gauss recorded in his diary that he had found a proof that every natural number is a sum of three triangular numbers. This is equivalent to the statement that every natural number of the form $8 n+3$ is represented as a sum of three squares. That only numbers not of the form $4^{a}(8 n+7)$ with $a$ and $n$ nonnegative integers can be represented as a sum of three squares was proved by Legendre in 1798 and independently by Gauss himself in 1801. Later in 1862 Liouville considered a more general type of ternary Diophantine problem. Namely, he determined all triples of positive integers $(\alpha, \beta, \gamma)$ for which the ternary sums $\alpha T_{x}+\beta T_{y}+\gamma T_{z}$ represent all positive integers. Here, $T_{x}$ denotes the polynomial $x(x+1) / 2$ in the variable $x$. For a more detailed historical account of subject, the readers are referred to [1] and [15]. Recently, Sun and his collaborators determined in [7, [12] and [13] all triples of positive integers $(\alpha, \beta, \gamma)$ for which the mixed sums of squares and triangular numbers of the forms $\alpha x^{2}+\beta y^{2}+\gamma T_{z}$ and $\alpha x^{2}+\beta T_{y}+\gamma T_{z}$ represent all positive integers.

We call a polynomial of the form $\alpha T_{x}+\beta T_{y}+\gamma T_{z}$ a ternary triangular form. A ternary triangular form is said to be almost universal if it represents all but finitely many positive integers. In [14 Sun conjectures some infinite families of ternary triangular forms to be almost universal. All the triangular forms on Sun's list have been studied by Kane [9, and for each of them he has either confirmed or disproved its almost universality. In their work [10, Theorem 1.17] Kane and Sun

Received by the editors August 28, 2008.

2000 Mathematics Subject Classification. Primary 11E12, 11E20.

The work of the second author was supported by the Korea Research Foundation Grant (KRF2008-314-C00004) funded by the Korean Government. 
give necessary and sufficient conditions on $(\alpha, \beta, \gamma)$ with $\operatorname{ord}_{2}(\alpha)=0 \leq \operatorname{ord}_{2}(\beta) \leq$ $\operatorname{ord}_{2}(\gamma)$ such that $\alpha T_{x}+\beta T_{y}+\gamma T_{z}$ is almost universal, except in the cases when

$$
\left(\operatorname{ord}_{2}(\beta), \operatorname{ord}_{2}(\gamma)\right)=(0,3),(1,4),(2,2) \quad \text { and } \operatorname{ord}_{2}(\beta)=3 \text { or } 4 .
$$

In these exceptional cases they give only sufficient conditions, but in 10 , Conjecture 1.19(ii)] they conjecture that those sufficient conditions are also necessary.

The main goal of this paper is to resolve Kane and Sun's conjecture. Our approach is similar to that of [10] in the sense that both rely heavily on the theory of primitive spinor exceptions of ternary quadratic forms. However, instead of using only diagonal quadratic forms derived from $\alpha T_{x}+\beta T_{y}+\gamma T_{z}$, we adopt the geometric language of quadratic spaces and lattices which is more flexible and hence allows us to use more fully the known results. In particular, the representations of a ternary triangular form $\alpha T_{x}+\beta T_{y}+\gamma T_{z}$ are the same as the representations of the ternary $\mathbb{Z}$-lattice $L$ associated to the quadratic form $\alpha x^{2}+\beta y^{2}+\gamma z^{2}$ satisfying some specific congruence conditions. This, in turn, is equivalent to the representations of a suitable coset of the sublattice $2 L$. We then consider the $\mathbb{Z}$-lattice $M$ generated by this coset and other auxiliary $\mathbb{Z}$-lattices, which are not necessarily diagonal, whose representations without congruence conditions will correspond to the representations of the original ternary triangular form.

Instead of giving the arguments just enough for proving Kane and Sun's conjecture, we provide the proof of a complete characterization of all positive almost universal triangular forms. Theorems 3.1 to 3.6 presented in Section 3 together effectively determine when a given triangular form $\alpha T_{x}+\beta T_{y}+\gamma T_{z}$ is almost universal. This not only is for the readers' convenience but also provides a much better understanding of the geometric setting we described above.

The subsequent discussion involves the computation of the spinor norms of local integral rotations and the relative spinor norm groups of primitive representations of integers by ternary quadratic forms. The readers can find all the relevant material and formulae in [4, [5, 6] and [8].

\section{Notation AND PRELIMINARIES}

Henceforth, the geometric language of quadratic spaces and lattices will be adopted, following [11. Any unexplained notation and terminology can be found there. All the $\mathbb{Z}$-lattices discussed below are positive definite. If $K$ is a $\mathbb{Z}$-lattice and $A$ is a symmetric matrix, we shall write " $K \cong A$ " if $A$ is the Gram matrix for $K$ with respect to some basis of $K$. The discriminant of $K$, denoted $d K$, is the determinant of $A$. An $n \times n$ diagonal matrix with $a_{1}, \ldots, a_{n}$ as the diagonal entries is written as $\left\langle a_{1}, \ldots, a_{n}\right\rangle$. The symbol $\theta$ always denotes the spinor norm map.

When we work with primitive spinor exception, we shall follow the notation introduced in 6. In particular, if $t$ is a primitive spinor exception of gen $(K)$ and $p$ is a prime, then $\theta^{*}\left(K_{p}, t\right)$ is the primitive relative spinor norm group of the $\mathbb{Z}_{p^{-}}$ lattice $K_{p}$. If $E$ is a quadratic extension of $\mathbb{Q}, N_{p}(E)$ denotes the group of local norms from $E_{\mathfrak{p}}$ to $\mathbb{Q}_{p}$, where $\mathfrak{p}$ is an extension of $p$ to $E$.

Let $a, b$ and $c$ be relatively prime positive odd integers, and let $r \leq s$ be nonnegative integers. The square-free part of $a b c$ is denoted by $\operatorname{sf}(a b c)$. Let $L$ be the $\mathbb{Z}$-lattice $\left\langle a, 2^{r} b, 2^{s} c\right\rangle$ with respect to the orthogonal basis $\left\{x_{1}, x_{2}, x_{3}\right\}$, and let $v:=x_{1}+x_{2}+x_{3}$. Then a simple algebraic computation reveals that a positive 
integer $n$ is represented by $a T_{x}+2^{r} b T_{y}+2^{s} c T_{z}$ if and only if $a+2^{r} b+2^{s} c+8 n$ is represented by the coset $v+2 L$.

Lemma 2.1. If $a T_{x}+2^{r} b T_{y}+2^{s} c T_{z}$ is almost universal, then $L_{p}$ represents all $p$-adic integers for every odd prime $p$. Consequently, we have

(1) $L_{p} \cong\langle 1,-1,-d L\rangle$ and $\theta\left(O^{+}\left(L_{p}\right)\right) \supseteq \mathbb{Z}_{p}^{\times}$for all odd primes $p$;

(2) $\mathbb{Q}_{2} L$ is anisotropic.

Proof. Suppose that $a T_{x}+2^{r} b T_{y}+2^{s} c T_{z}$ is almost universal. Then $v+2 L$ represents all but finitely many integers of the form $a+2^{r} b+2^{s} c+8 n$. Let $p$ be an odd prime. Since $2 v \in 2 L, L_{p}=v+L_{p}$. Therefore, $L_{p}$ must represent all $p$-adic integers. In particular $L_{p}$ must be isotropic. All these together show that $L_{p} \cong\langle 1,-1,-d L\rangle$. The second assertion in (1) is [11, 92:5].

For (2), note that for every place $p$ (including the case $p=\infty$ ), $\mathbb{Q}_{p} L$ is isotropic if and only if the Hasse invariant of $\mathbb{Q}_{p} L$ is equal to $(-1,-1)_{v}$ [11, 58:7a]. By the Hilbert Reciprocity Law it follows that the number of places $p$ over which $\mathbb{Q}_{p} L$ is anisotropic is even. Since $\mathbb{R} L$ is anisotropic, $\mathbb{Q}_{2} L$ must be anisotropic as well.

The condition that $L_{p}$ represents all $p$-adic integers for every odd prime $p$ can be stated in the following more elementary manner:

(i) $a, b, c$ are pairwise relatively prime; and (ii) if an odd prime $p$ divides one of $a, 2^{r} b$ or $2^{s} c$, then the negative of the product of the other two is a square modulo $p$.

This is precisely the condition described in [10, Lemma 3.1].

Let $M$ be the $\mathbb{Z}$-lattice $\mathbb{Z} v+2 L$. Its Gram matrix relative to the basis $\left\{2 x_{1}, 2 x_{2}, v\right\}$ is

$$
\left(\begin{array}{ccc}
4 a & 0 & 2 a \\
0 & 2^{r+2} b & 2^{r+1} b \\
2 a & 2^{r+1} b & a+2^{r} b+2^{s} c
\end{array}\right)
$$

and its discriminant is $2^{r+s+4} a b c$. Let $P$ be the binary sublattice $\mathbb{Z} 2 x_{2}+\mathbb{Z} v$, which is isometric to

$$
\left(\begin{array}{cc}
2^{r+2} b & 2^{r+1} b \\
2^{r+1} b & a+2^{r} b+2^{s} c
\end{array}\right)
$$

with discriminant $2^{r+2} b\left(a+2^{s} c\right)$.

Lemma 2.2. Suppose that $L_{p}$ represents all p-adic integers for every odd prime $p$. Then

(1) every positive integer of the form $a+2^{r} b+2^{s} c+8 n$ is represented primitively by $\operatorname{gen}(M)$;

(2) ift is a primitive spinor exception of $M$, then $\mathbb{Q}(\sqrt{-t d M})$ is either $\mathbb{Q}(\sqrt{-1})$ or $\mathbb{Q}(\sqrt{-2})$.

Proof. (1) This is obvious if $a+2^{r} b+2^{s} c$ is odd. Suppose that it is even. Then $0=r<s$ and, since $L_{2}$ is anisotropic, $a+b \equiv 2 \bmod 4$ or $\equiv 4 \bmod 8$.

Suppose that $a+b \equiv 2 \bmod 4$. When $s \geq 2$, then $\operatorname{ord}_{2}\left(a+b+2^{s} c+8 n\right)=1$ for all $n$ and $P_{2}$ is a proper 2-modular $\mathbb{Z}_{2}$-lattice which represents $a+b+2^{s} c$. Therefore, $P_{2}$ also represents $a+b+2^{s} c+8 n$ for all $n$. If $s=1,-d P=-4 b(a+2 c)$, which cannot be a square in $\mathbb{Z}_{2}$. Therefore, $a+b+2 c \equiv 4 \bmod 8$. This implies that $P_{2} \cong 2 A(2,2)$, which certainly represents all integers of the form $a+b+2 c+8 n$. 
Suppose now that $a+b \equiv 4 \bmod 8$. Then $s$ is odd since $L_{2}$ is anisotropic. If $s=1$, then $P_{2}$ is proper 2-modular; otherwise $P_{2} \cong 2 A(2,2)$. In either case, the proof can proceed as is in the last paragraph.

(2) Let $E:=\mathbb{Q}(\sqrt{-t d M})$. For all odd primes $p$, we have $\mathbb{Z}_{p}^{\times} \subseteq \theta\left(O^{+}\left(M_{p}\right)\right) \subseteq$ $N_{p}(E)$. Therefore, $E$ must be an imaginary quadratic extension unramified outside 2. As a result, $E=\mathbb{Q}(\sqrt{-1})$ or $\mathbb{Q}(\sqrt{-2})$.

Lemma 2.3. Suppose that $L_{p}$ represents all $p$-adic integers for every odd prime $p$. If ord $_{2}\left(a+2^{r} b+2^{s} c\right) \leq 1$ and $a+2^{r} b+2^{s} c+8 n$ is not a primitive spinor exception of $\operatorname{gen}(M)$ for all $n \geq 0$, then $a T_{x}+2^{r} b T_{y}+2^{s} c T_{z}$ is almost universal.

Proof. Let $m$ be a positive integer of the form $a+2^{r} b+2^{s} c+8 n$. Since $\operatorname{spn}(M)$ represents $m$ primitively, $M$ represents $m$ primitively if $m$ is sufficiently large 2, Theorem 1]. But any representation of $m$ by $M$ must lie in $v+2 L$. Thus $a T_{x}+$ $2^{r} b T_{y}+2^{s} c T_{z}$ is almost universal.

\section{MAin RESUlts}

We continue to assume that $a, b, c$ are relatively prime positive odd integers and $r \leq s$ are nonnegative integers. Theorems 3.1 to 3.6 below determine exactly all the triples $\left(a, 2^{r} b, 2^{s} c\right)$ for which $a T_{x}+2^{r} b T_{y}+2^{s} c T_{z}$ are almost universal. There is no doubt that one can forge a single theorem by combining all six together. But we refrained from doing so since the end result would have been complicated and less transparent to the reader.

Theorem 3.1. Suppose that $a+b \equiv 2 \bmod 4$. Then $a T_{x}+b T_{y}+2^{s} c T_{z}$ is almost universal if and only if $a x^{2}+b y^{2}+2^{s} c z^{2}$ represents all $p$-adic integers over $\mathbb{Z}_{p}$ for every odd prime $p$ and one of the following holds:

(1) $s$ is even or $s=1$,

(2) $\operatorname{sf}(a b c)$ is divisible by a prime congruent to $3 \bmod 4$,

(3) $a b \not \equiv 1 \bmod 8$,

(4) $\left(2 \operatorname{sf}(a b c)-\left(a+b+2^{s} c\right)\right) / 8$ is represented by $a T_{x}+b T_{y}+2^{s} c T_{z}$.

Proof. Suppose that $a x^{2}+b y^{2}+2^{s} c z^{2}$ represents all $p$-adic integers for every odd prime $p$. Thus $M_{2}$, as a sublattice of $L_{2}$, is anisotropic. Therefore $P_{2}$ is also anisotropic and hence $-2^{2} b\left(a+2^{s} c\right)$ is not a square (or, equivalently, $a+b+2^{s} c \not \equiv 0$ $\bmod 8$ ) when $s>1$.

If $s=0, \mathbb{Z}_{2} v$ splits $M_{2}$ and its orthogonal complement is an improper modular lattice; hence $\theta\left(O^{+}\left(M_{2}\right)\right) \supseteq \mathbb{Z}_{2}^{\times}$. Together with Lemma 2.1(1) we deduce that gen $(M)$ has only one spinor genus. By Lemma 2.3, $a T_{x}+b T_{y}+c T_{z}$ is almost universal.

When $s=1$, Lemma 2.3 cannot apply even though one can show that gen $(M)$ still has only spinor genus. Consider the $\mathbb{Z}$-lattice $R$ with $R_{p}=M_{p}$ for all odd primes $p$ and

$$
R_{2}=\mathbb{Z}_{2} v+\mathbb{Z}_{2} 2 x_{3}+\mathbb{Z}_{2}\left(2 b x_{1}-2 a x_{2}\right) \cong\left(\begin{array}{cc}
a+b+2 c & 4 c \\
4 c & 8 c
\end{array}\right) \perp\langle 4 a b(a+b)\rangle .
$$

Note that $R \subseteq M$ and

$$
R_{2}^{1 / 4} \cong\left(\begin{array}{cc}
\frac{a+b+2 c}{4} & c \\
c & 2 c
\end{array}\right) \perp\langle a b(a+b)\rangle .
$$


Since $(a+b+2 c) / 4$ is odd, $\left(\begin{array}{cc}\frac{a+b+2 c}{4} & c \\ c & 2 c\end{array}\right)$ is a binary proper unimodular $\mathbb{Z}_{2}$-lattice. This, together with the fact that $a+b \equiv 2 \bmod 4$, shows that $R_{2}^{1 / 4}$ primitively represents all odd integers. For any odd integer $t$, the extension $\mathbb{Q}_{2}\left(\sqrt{-t d R^{1 / 4}}\right) / \mathbb{Q}_{2}$ is ramified and $\operatorname{ord}_{2}\left(-t d R^{1 / 4}\right)$ is odd. By [6. Theorem 2(c)], $\theta\left(O^{+}\left(R_{2}^{1 / 4}\right)\right)$ is not contained in the norm group of $\mathbb{Q}_{2}\left(\sqrt{-t d R^{1 / 4}}\right)$. Therefore, $t$ is never a primitive spinor exception of $\operatorname{gen}\left(R^{1 / 4}\right)$. By [2, Theorem 1], $R$ represents all but finitely many positive integers of the form $a+b+2 c+8 n$. But any representation of those integers in $R$ must lie inside $v+2 L$. This shows that $a T_{x}+b T y+2 c T_{z}$ is almost universal.

Suppose that $s \geq 2$. In particular, $\operatorname{ord}_{2}\left(a+b+2^{s} c\right)=1$. It is more convenient to work with the lattice $G:=M^{1 / 2}$. Note that $d G=2^{s+1} a b c$ and

$$
G_{2} \cong\left\langle\epsilon, \epsilon b\left(a+2^{s} c\right)\right\rangle \perp 2^{s+1}\left\langle a c\left(a+2^{s} c\right)\right\rangle, \quad \epsilon:=\left(a+b+2^{s} c\right) / 2 .
$$

We first determine the conditions for which gen $(G)$ does not have an odd primitive spinor exception and hence Lemma 2.3 applies. Suppose that $t$ is an odd primitive spinor exception of $\operatorname{gen}(G)$. Let $E$ be the field $\mathbb{Q}(\sqrt{-t d G})$. Since $\theta\left(O^{+}\left(G_{p}\right)\right) \supseteq \mathbb{Z}_{p}^{\times}$ for all odd primes $p, E$ is either $\mathbb{Q}(\sqrt{-1})$ or $\mathbb{Q}(\sqrt{-2})$ (see the proof of Lemma $2.2(2))$. When $s$ is even, $\operatorname{ord}_{2}(d G)=s+1$ is odd, and so $E=\mathbb{Q}(\sqrt{-2})$. But by 6 , Theorem $2(\mathrm{c})$ ], since $r=0, \theta\left(O^{+}\left(G_{2}\right)\right) \nsubseteq N_{2}(E)$. Hence gen $(G)$ does not have any odd primitive spinor exception when $s$ is even.

Now, let us assume that $s$ is odd. Then $E=\mathbb{Q}(\sqrt{-1})$, and so $t=m^{2} \operatorname{sf}(a b c)$ for some odd integer $m$. Let $p$ be a prime congruent to $3 \bmod 4$. By [6, Theorem $1(\mathrm{a})]$, either $\operatorname{ord}_{p}(\operatorname{sf}(a b c))=0$ or $p \nmid t$. Thus every prime divisor of $\operatorname{sf}(a b c)$ must be congruent to $1 \bmod 4$. At the prime 2 , we have

$$
G_{2} \cong\langle\epsilon, \epsilon a b\rangle \perp 2^{s+1}\langle c\rangle .
$$

From [8, Proposition B] we know that $\theta\left(O^{+}\left(G_{2}\right)\right)$ contains $\left\{x \in \mathbb{Q}_{2}^{\times}:(x,-a b)_{2}=\right.$ $1\}$. But since $\theta\left(O^{+}\left(G_{2}\right)\right)$ is a subgroup of $N_{2}(E)$, we must have

$$
\theta\left(O^{+}\left(G_{2}\right)\right)=\left\{x \in \mathbb{Q}_{2}^{\times}:(x,-1)_{2}=1\right\}
$$

and $a b \equiv 1 \bmod 8$. Therefore if either (2) or (3) is satisfied, then $\operatorname{gen}(G)$ does not have any odd primitive spinor exception.

We claim that if $a b \equiv 1 \bmod 8$ and every prime divisor of $\operatorname{sf}(a b c)$ is congruent to $1 \bmod 4$, then $\operatorname{sf}(a b c)$ is a primitive spinor exception of $\operatorname{gen}(G)$. Under these conditions we have $\operatorname{sf}(a b c) \equiv 1 \equiv \epsilon \bmod 4$. Thus $\operatorname{sf}(a b c)$ is represented primitively by $\operatorname{gen}(G)$. The field $\mathbb{Q}(\sqrt{-\operatorname{sf}(a b c) d G})$, still denoted by $E$ since no confusion will arise, is just $\mathbb{Q}(\sqrt{-1})$. When $p$ is an odd prime, it follows easily from [6, Theorem 1$]$ that $\theta\left(O^{+}\left(G_{p}\right)\right) \subseteq N_{p}(E)$ and $\theta^{*}\left(G_{p}, \operatorname{sf}(a b c)\right)=N_{p}(E)$. For the prime 2 , we need to look at [6, Theorem 2(b)]. It is clear that $\theta\left(O^{+}\left(G_{2}\right)\right)=N_{2}(E)$. The lattice $K$ in [6. Theorem 2(b)] is $\left\langle 2^{-2} \epsilon, \epsilon a b, 2^{s+1} c\right\rangle$; hence $\theta\left(O^{+}(K)\right) \subseteq\{1,2,5,10\} \mathbb{Q}_{2}^{\times 2}$ by [4, Theorem 2.7], and so $\theta\left(O^{+}(K)\right) \subseteq N_{2}(E)$. The lattice $K^{\prime}$ in [6, Theorem 2(b)] is just $G_{2}$ itself; thus $\theta\left(O^{+}\left(K^{\prime}\right)\right) \subseteq N_{2}(E)$. Clearly, $2^{s+1} \nmid \operatorname{sf}(a b c)$. Therefore, $\theta^{*}\left(G_{2}, \operatorname{sf}(a b c)\right)=N_{2}(E)$. This proves our claim.

Now suppose that conditions (1), (2) and (3) are not satisfied. If $\operatorname{sf}(a b c)$ is not represented by $G$, then by [3, Theorem 1] there are infinitely many odd primes $p$ such that $\operatorname{sf}(a b c) p^{2}$ is not represented primitively by $G$. For each of these $p$, we have

$$
\operatorname{sf}(a b c) p^{2} \equiv \operatorname{sf}(a b c) \equiv 1 \equiv \epsilon \quad \bmod 4
$$


Therefore, for infinitely many such $p, n:=\left(2 \operatorname{sf}(a b c) p^{2}-\left(a+b+2^{s} c\right)\right) / 8$ is a positive integer and $a+b+2^{s} c+8 n$ is not represented primitively by $M$. But this is the same as saying that $a+b+2^{s} c+8 n$ is not represented by $v+2 L$. So $a T_{x}+b T_{y}+2^{s} c T_{z}$ is not almost universal.

Conversely, suppose that $\operatorname{sf}(a b c)$ is represented by $G$. If $a+b+2^{s} c+4 n$ is not a primitive spinor exception of $\operatorname{gen}(G)$, then $a+b+2^{s} c+4 n$ is represented by $G$ if $n$ is sufficiently large. Otherwise, $a+b+2^{s} c+4 n$ must be a square multiple of $\operatorname{sf}(a b c)$ and hence $a+b+2^{s} c+4 n$ is represented by $G$. We can now conclude that $a+b+2^{s} c+8 n$ is represented by $M$ for almost all $n$. But again any representation of $a+b+2^{s} c+8 n$ must be in $v+2 L$; hence $a T_{x}+b T_{y}+2^{s} c T_{z}$ is almost universal. This completes the proof of the theorem.

The next case we consider is when $r=0$ and $a+b \equiv 0 \bmod 4$.

Theorem 3.2. Suppose that $a+b \equiv 0 \bmod 4$.

(a) If $a+b \equiv 0 \bmod 8$, then $a T_{x}+b T_{y}+2^{s} c T_{z}$ is not almost universal.

(b) If $a+b \equiv 4 \bmod 8$, then $a T_{x}+b T_{y}+2^{s} c T_{z}$ is almost universal if and only if $a x^{2}+b y^{2}+2^{s} c z^{2}$ represents all $p$-adic integers over $\mathbb{Z}_{p}$ for every odd prime $p$ and $s$ is odd.

Proof. (a) This is clear since $L_{2}$ is isotropic if $a+b \equiv 0 \bmod 8$.

(b) We assume throughout that $a x^{2}+b y^{2}+2^{s} c z^{2}$ represents odd $p$-adic integers for every odd prime $p$. Since $a+b \equiv 4 \bmod 8, a b \equiv 3 \bmod 8$. Thus $a T_{x}+b T_{y}+2^{s} c T_{z}$ is not almost universal if $s$ is even, since in this case $L_{2}$ is isotropic.

Suppose that $s=1$. Since once again $a b \equiv 3 \bmod 8, \operatorname{sf}(a b c)$ must be divisible by a prime that is congruent to $3 \bmod 8$. This shows that $\operatorname{gen}(G)$ cannot have any odd primitive spinor exception, where $G=M^{1 / 2}$ as is in the proof of the previous theorem. We then apply Lemma 2.3 to show that $a T_{x}+b T_{y}+2^{s} c T_{z}$ is almost universal.

Now, let us suppose that $s \geq 3$ and $s$ is odd. Consider the sublattice $R$ of $M$ defined by setting $R_{p}=M_{p}$ for all odd primes $p$ and $R_{2}=\mathbb{Z}_{2} v+\mathbb{Z}_{2} 4 x_{1}+$ $\mathbb{Z}_{2}\left(2^{s+1} c x_{2}-2 b x_{3}\right)$. It is not hard to see that

$$
R_{2}^{1 / 4} \cong\left(\begin{array}{cc}
\frac{a+b+2^{s} c}{4} & a \\
a & 4 a
\end{array}\right) \perp\left\langle 2^{s} c\right\rangle .
$$

One can show that gen $\left(R^{1 / 4}\right)$ primitively represents all positive odd integers, and by [6. Theorem $2(\mathrm{c})$ ] it follows that gen $\left(R^{1 / 4}\right)$ does not have any odd primitive spinor exception. Therefore, $R$ primitively represents all sufficiently large integers of the form $a+b+2^{s} c+8 n$, and so $a T_{x}+b T_{y}+2^{s} c T_{z}$ is almost universal.

We now proceed to the cases in which $0<r<s$. For technical reasons, we treat the cases $3 \leq r$ and $r=1$ or 2 separately. Over $\mathbb{Z}_{2}$,

$$
M_{2} \cong\langle\eta\rangle \perp 2^{r+2}\left\langle a b\left(a+2^{r} b\right)\right\rangle \perp 2^{s+2}\left\langle c\left(a+2^{r} b\right) \eta\right\rangle,
$$

where $\eta:=a+2^{r} b+2^{s} c$. If $(s, r)=(1,2),(2,3)$ or $(2,5)$, then $M_{2}$ is of type $E$ (see [5, page 531] and [4, Theorem 2.2]) and $\theta\left(O^{+}\left(M_{2}\right)\right)=\mathbb{Q}_{2}^{\times}$. Otherwise, $\theta\left(O^{+}\left(M_{2}\right)\right)$ is computed as follows. Let

$U:=\langle 1\rangle \perp 2^{r+2}\left\langle a b\left(a+2^{r} b\right) \eta\right\rangle \quad$ and $\quad W:=2^{r+2}\left\langle a b\left(a+2^{r} b\right) \eta\right\rangle \perp\left\langle 2^{s+2}\left\langle c\left(a+2^{r} b\right)\right\rangle\right.$. 
Also, let $\mathcal{P}(U)$ be the set of primitive anisotropic vectors in $U$ whose associate symmetries are in $O(U)$. The set $\mathcal{P}(W)$ is defined similarly. Then $\theta\left(O^{+}\left(M_{2}\right)\right)=$ $Q(\mathcal{P}(U)) Q(\mathcal{P}(W)) \mathbb{Q}_{2}^{\times 2}$ [4, Theorem 2.7]. Note that $Q(\mathcal{P}(U)) \mathbb{Q}_{2}^{\times 2}=\theta\left(O^{+}(U)\right)$ and $Q(\mathcal{P}(W))=2^{r+2} a b\left(a+2^{r} b\right) \eta \theta\left(O^{+}(W)\right)$. The formulae for $\theta\left(O^{+}(U)\right)$ and $\theta\left(O^{+}(W)\right)$ are available in [4, 1.9]. Define an integer $\delta$ by

$$
\delta:= \begin{cases}1 & \text { if } r+s \text { is even } \\ 2 & \text { otherwise. }\end{cases}
$$

Theorem 3.3. Suppose that $3 \leq r<s$. Then $a T_{x}+2^{r} b T_{y}+2^{s} c T_{z}$ is almost universal if and only if $a x^{2}+2^{r} b y^{2}+2^{s} c z^{2}$ represents all $p$-adic integers over $\mathbb{Z}_{p}$ for every odd prime $p$ and one of the following holds:

(1) $\operatorname{sf}(a b c)$ is divisible by a prime $p$ satisfying $\left(\frac{-\delta}{p}\right)=-1$,

(2) $b c \not \equiv 1 \bmod 8$,

(3) $\left(\operatorname{sf}(a b c)-\left(a+2^{r} b+2^{s} c\right)\right) / 8$ is represented by $a T_{x}+2^{r} b T_{y}+2^{s} c T_{z}$.

Proof. Suppose that $a x^{2}+2^{r} b y^{2}+2^{s} c z^{2}$ represents all $p$-adic integers over $\mathbb{Z}_{p}$ for every odd prime $p$. The proof is a case-by-case analysis according to $s-r=1,2,3,4$ or $\geq 5$. However, since all these cases follow the same line of argument, we shall provide details only for the cases $s-r=1$ or 3 and indicate at the end what is needed to be changed in the proofs for the other cases.

Suppose that $s-r=1$ or 3 . If $t$ is an odd primitive spinor exception of $\operatorname{gen}(M)$, then $E:=\mathbb{Q}(\sqrt{-t d M})$ must be $\mathbb{Q}(\sqrt{-2})$. Moreover, $t=m^{2} \operatorname{sf}(a b c)$ for some odd integer $m$. This implies that $a b c \equiv a \bmod 8$; that is, $b c \equiv 1 \bmod 8$. It also follows from [6, Theorem 1] that all prime divisors of $\operatorname{sf}(a b c)$ must be congruent to 1 or 3 mod 8 (those are the primes $p$ such that $\left(\frac{-2}{p}\right)=1$ ). At this point we have shown that $\operatorname{gen}(M)$ does not have any primitive spinor exception when either (1) or (2) holds.

Now let us suppose that both (1) and (2) do not hold. We claim that $\operatorname{sf}(a b c)$ is an odd primitive spinor exception of $\operatorname{gen}(M)$. It is clear from [6, Theorem 1] that $\theta\left(O^{+}\left(M_{p}\right)\right) \subseteq N_{p}(E)$ and $\theta^{*}\left(M_{p}, \operatorname{sf}(a b c)\right)=N_{p}(E)$ for all odd primes $p$. Over $\mathbb{Z}_{2}$,

$$
U \cong\langle 1\rangle \perp 2^{r+2}\langle a b\rangle, \quad W \cong 2^{r+2} a b\left(\langle 1\rangle \perp 2^{s-r}\langle 1\rangle\right) .
$$

Since $r+2 \geq 5$ and $s-r=1$ or 3 , we have

$$
Q(\mathcal{P}(U))=\left\{1,2^{r+2} a b\right\} \mathbb{Q}_{2}^{\times 2}, \quad Q(\mathcal{P}(W))=2^{r+2} a b\{1,2,3,6\} \mathbb{Q}_{2}^{\times 2} .
$$

Thus $\theta\left(O^{+}\left(M_{2}\right)\right)=N_{2}(E)$. Now, by [6, Theorem 2(c)] we have $\theta^{*}\left(M_{2}, \operatorname{sf}(a b c)\right)=$ $N_{2}(E)$. Therefore, $\operatorname{sf}(a b c)$ is a primitive spinor exception of gen $(M)$.

The final step is to show that if both (1) and (2) do not hold, then $a T_{x}+2^{r} b T_{y}+$ $2^{s} c T_{z}$ is almost universal if and only if (3) holds. This can be done by the same argument used in the proof of Theorem 3.1.

For the rest of the cases, there are the following slight changes that need to be made. First, the field $E=\mathbb{Q}(\sqrt{-t d M})$ is $\mathbb{Q}(\sqrt{-1})$ if $s-r$ is even, and $\mathbb{Q}(\sqrt{-2})$ if $s-r$ is odd. This explains the appearance of the quantity $\delta$ in the statement of the theorem. Second, as $s-r$ changes the set $Q(\mathcal{P}(W))$ changes as follows [4, 1.9] 
(assuming $b c \equiv 1 \bmod 8$ ):

$$
Q(\mathcal{P}(W))= \begin{cases}2^{r+2} a b\{1,5\} \mathbb{Q}_{2}^{\times 2} & \text { if } s-r=2 \text { or } 4, \\ 2^{r+2} a b\left\{1,2^{s-r}\right\} \mathbb{Q}_{2}^{\times 2} & \text { if } s-r \geq 5 .\end{cases}
$$

We therefore still have $\theta\left(O^{+}\left(M_{2}\right)\right) \subseteq N_{2}(E)$ in all cases.

Theorem 3.4. The triangular form $a T_{x}+2 b T_{y}+2^{s} c T_{z}, s>1$, is almost universal if and only if ax $x^{2}+2 b y^{2}+2^{s} c z^{2}$ represents all p-adic integers over $\mathbb{Z}_{p}$ for every odd prime $p$ and one of the following holds:

(1) $s$ is odd or $s=2$,

(2) $\operatorname{sf}(a b c)$ is divisible by a prime congruent to 5 or $7 \bmod 8$,

(3) $a b c \not \equiv a+2 b \bmod 8$,

(4) $\left(\operatorname{sf}(a b c)-\left(a+2 b+2^{s} c\right)\right) / 8$ is represented by $a T_{x}+2 b T_{y}+2^{s} c T_{z}$.

Theorem 3.5. The triangular form $a T_{x}+4 b T_{y}+2^{s} c T_{z}, s>2$, is almost universal if and only if a $x^{2}+4 b y^{2}+2^{s} c z^{2}$ represents all $p$-adic integers over $\mathbb{Z}_{p}$ for every odd prime $p$ and one of the following holds:

(1) $s$ is odd,

(2) $\operatorname{sf}(a b c)$ is divisible by a prime congruent to $3 \bmod 4$,

(3) $a b c \not \equiv a+4 b \bmod 8$,

(4) $\left(\operatorname{sf}(a b c)-\left(a+4 b+2^{s} c\right)\right) / 8$ is represented by $a T_{x}+4 b T_{y}+2^{s} c T_{z}$.

Theorem 3.6. The triangular form $a T_{x}+2^{r} b T_{y}+2^{r} c T_{z}$ is almost universal if and only if ax $x^{2}+2^{r} b y^{2}+2^{r} c z^{2}$ represents all $p$-adic integers over $\mathbb{Z}_{p}$ for every odd prime $p$ and one of the following holds:

(1) $r=0$ or 1 ,

(2) $\operatorname{sf}(a b c)$ is divisible by a prime congruent to $3 \bmod 4$,

(3) $b c \neq \equiv 1(\bmod 8)$,

(4) $\left(\operatorname{sf}(a b c)-\left(a+2^{r} b+2^{r} c\right)\right) / 8$ is represented by $a T_{x}+2^{r} b T_{y}+2^{r} c T_{z}$.

The last three theorems cover all the remaining cases. Their proofs use the same idea and method we have been using thus far, except that different formula 1 for $\theta\left(O^{+}\left(M_{2}\right)\right)$ are used in different cases. Instead of burdening the readers with repetitive arguments, we simply outline the steps as follows. Once again, we assume that the triangular form in question represents all $p$-adic integers over $\mathbb{Z}_{p}$ for every odd prime $p$. Under this assumption it can be shown that $\operatorname{gen}(M)$ does not have any odd primitive spinor exception if one of the first three conditions holds; hence Lemma 2.3 applies. Otherwise, we show that the corresponding triangular form is almost universal if and only if (4) holds.

The following is conjectured by Kane and Sun in [10, Conjecture 1.19(ii)], which completes their classification of almost universal positive triangular forms.

Corollary 3.7. Suppose that $r \in\{3,4\}$ or $(r, s)=(2,2),(0,3)$ or $(1,3)$, and that $a x^{2}+2^{r} b y^{2}+2^{s} c z^{2}$ represents all $p$-adic integers over $\mathbb{Z}_{p}$ for every odd prime $p$.

\footnotetext{
${ }^{1}$ John Hsia communicated to the first author that [4. Theorem 3.14] and even its corrected version [5, 1.2] need modification. The beginning of the fourth line of [5, 1.2] should be "all $2^{r_{i}} M_{i}$ have the same order...," and one should change Case (3)(iii) in [5] 1.2] to " $r_{j+1}-r_{j} \geq 4$, or $r_{j+1}-r_{j}=2$ with $\operatorname{rank}\left(L_{j}\right)=\operatorname{rank}\left(L_{j+1}\right)=1$ and $\operatorname{det}\left(2^{r_{j}} L_{j} \perp 2^{r_{j+1}} L_{j+1}\right) \operatorname{det}\left(2^{r_{i}} L_{i_{0}}\right) \in$ $\{1, \Delta\}$, for $i=1, \ldots, t-1 . "$
} 
Then $a T_{x}+2^{r} b T_{y}+2^{s} c T_{z}$ is not almost universal if the following three conditions hold:

(1) $4 \nmid a+2^{r} b+2^{s} c$ and $\operatorname{sf}(a b c) \equiv 2^{-v}\left(a+2^{r} b+2^{s} c\right) \bmod 2^{3-v}$, where $v=$ $\operatorname{ord}_{2}\left(a+2^{r} b+2^{s} c\right)<2$.

(2) All prime divisors of $\operatorname{sf}(a b c)$ are congruent to 1 modulo 4 if $\operatorname{sf}\left(2^{r+s} a b c\right) \equiv$ $a+2^{r} b+2^{s} c \bmod 2$, and congruent to 1 or 3 modulo 8 otherwise.

(3) $a x^{2}+2^{r} b y^{2}+2^{s} c z^{2}=2^{v} \operatorname{sf}(a b c)$ has no integral solutions with $x, y$, $z$ all odd.

Proof. Note that condition (3) is equivalent to saying that $\left(2^{v} \operatorname{sf}(a b c)-\left(a+2^{r} b+\right.\right.$ $\left.\left.2^{s} c\right)\right) / 8$ is not represented by $a T_{x}+2^{r} b T_{y}+2^{s} c T_{z}$.

We first treat the case $(r, s)=(0,3)$. Suppose $a+b \equiv 2 \bmod 4$. Then $a+b=2 t$ with $t$ odd. Conditions (1) and (2) together imply that $t \equiv 1 \bmod 4$, which means that $a \equiv b \bmod 8$. By Theorem 3.1, $a T_{x}+2^{r} b T_{y}+2^{s} c T_{z}$ is not almost universal. In the case of $a+b \equiv 0 \bmod 4$, condition (1) is never satisfied, and hence the corollary is just a consequence of Theorem 3.2 .

The other cases can be verified similarly. Indeed, Theorem 3.4 and Theorem 3.6 deal with the cases $(r, s)=(1,4)$ and $(r, s)=(2,2)$, respectively, while Theorem 3.3 resolves the case when $r=3$ or 4 .

We conclude by exhibiting a few examples which are direct consequences of the main theorems.

Example 3.8. The triangular form $T_{x}+T_{y}+2^{s} c T_{z}$ is almost universal if and only if every prime divisor of $c$ is congruent to $1 \bmod 4$ and $s$ is 1 or even.

Example 3.9. The triangular form $T_{x}+2 T_{y}+2^{s} c T_{z}$ is almost universal if and only if every prime divisor of $c$ is congruent to 1 or $3 \bmod 8$ and one of the following holds:

(1) $s$ is 2 or odd,

(2) $s$ is even and $c \equiv 1 \bmod 8$.

Example 3.10. The triangular form $T_{x}+2^{r} T_{y}+2^{s} T_{z}, 0 \leq r \leq s$, is almost universal if and only if one of the following holds:

(1) $r=0$ and $s$ is 1 or even,

(2) $r=1$,

(3) $r=2$ and $s \geq 3$.

Remark 3.11. In Corollaries 1.16 and 1.18 of [10, Kane and Sun give necessary and sufficient conditions on $m$ for which $T_{x}+2^{\epsilon} T_{y}+m T_{z}$ is almost universal when $\epsilon=0$ or 1 and $\operatorname{ord}_{2}(m) \neq 3+\epsilon$. Our Examples 3.8 and 3.9 subsume their results and include the untreated case $\operatorname{ord}_{2}(m)=3+\epsilon$.

\section{ACKnowledgement}

The authors thank the referee for valuable comments and suggestions.

\section{REFERENCES}

[1] L.E. Dickson, History of the Theory of Numbers, Vol. II, AMS Chelsea Publ., 1999. MR 0245500 (39:6807b)

[2] W. Duke, and R. Schulze-Pillot, Representation of integers by positive ternary quadratic forms and equidistribution of lattice points on ellipsoids, Invent. Math. 99 (1990), no. 1, 49-57. MR:1029390 (90m:11051) 
[3] A.G. Earnest, Representation of spinor exceptional integers by ternary quadratic forms, Nagoya Math. J. 93 (1984), 27-38. MR738916 (85j:11042)

[4] A.G. Earnest and J.S. Hsia, Spinor norms of local integral rotations. II, Pacific J. Math. 61 (1975), no. 1, 71-86. MR0404142(53:7946)

[5] A.G. Earnest and J.S. Hsia, Spinor genera under field extensions. II. 2 unramified in the bottom field, Amer. J. Math. 100 (1978), no. 3, 523-528. MR0491488 (58:10731a)

[6] A.G. Earnest, J.S. Hsia and D.C. Hung, Primitive representations by spinor genera of ternary quadratic forms, J. London Math. Soc. (2) 50 (1994), no. 2, 222-230. MR1291733(95k:11044)

[7] S. Guo, H. Pan, Z.W. Sun, Mixed sums of squares and triangular numbers. II, Integers 7 (2007), \#A5, 5 pp. (electronic). MR:2373118(2008m:11078)

[8] J.S. Hsia, Spinor norms of local integral rotations. I, Pacific J. Math. 57 (1975), no. 1, 199-206. MR0374029 (51:10229)

[9] B. Kane, On two conjectures about mixed sums of squares and triangular numbers, J. Combinatorics and Number Theory 1 (2009), no. 1, 77-90.

[10] B. Kane and Z.W. Sun, On almost universal mixed sums of squares and triangular numbers, preprint arXiv:0808.2761.

[11] O.T. O'Meara, Introduction to quadratic forms, Springer-Verlag, New York, 1963. MR0152507 (27:2485)

[12] B.-K. Oh and Z.W. Sun, Mixed sums of squares and triangular numbers. III, J. Number Theory 129 (2009), 964-969.

[13] Z.W. Sun, Mixed sums of squares and triangular numbers, Acta Arith. 127 (2007), no. 2, 103-113. MR2289977 (2007m:11052)

[14] Z.W. Sun, A message to number theory mailing list, April 27, 2008. http://listserv.nodak.edu/cgi-bin/wa.exe?A2 $=$ ind0804\&L=nmbrthry\&T $=0 \& \mathrm{P}=1670$.

[15] A. Weil, Number Theory: An approach through history from Hammurapi to Legendre, Birkhäuser Boston, 1984. MR734177 (85c:01004)

Department of Mathematics and Computer Science, Wesleyan University, MiddleTOWn, CONNECTICUT 06459

E-mail address: wkchan@wesleyan.edu

Department of Mathematical Sciences, Seoul National University, Seoul 151-747, Korea

E-mail address: bkoh@math.snu.ac.kr 